\title{
PSS DESIGN INNOVATION: PROTOTYPING IN PRACTICE
}

\author{
R. Ruvald ${ }^{凶}$, A. Larsson, C. Johansson Askling and A. Bertoni \\ Blekinge Institute of Technology, Sweden \\ $\bowtie$ ryan.ruvald@bth.se
}

\begin{abstract}
Heavy equipment manufacturers recognise an opportunity to realise customer value gains through offering new Product-Service Systems. Such transition implies a radical shift in how new systems are designed. Based on a set of interviews the paper investigates how radical PSS innovation can be enabled by the use of physical prototypes as boundary object to navigate early PSS design ambiguity. On such basis, suggestions for augmenting existing support tools are made in relation to the existing literature.
\end{abstract}

Keywords: product-service systems (PSS), prototyping, design thinking, design support system

\section{Introduction}

Equipment manufacturers supplying incumbent construction industries (e.g. construction, quarry and mining operations) are currently operating in saturated markets where product differentiation is relatively low between manufacturers. To create substantial separation manufacturers are attempting to "disrupt" themselves by employing 'radical' innovation strategies aimed at providing exponential increases in efficiency, sustainability, performance, safety or whatever the customer defines as their core values (Norman et al., 2013). Achieving substantial gains across these core values cannot be realized by pure product innovation or the addition of aftermarket service contracts alone, but rather addressing multiple "ilities" in the development process (Bertoni et al., 2019). A framework equipment manufacturers are increasingly exploring to achieve this radical innovation effort is the coupling of new and/or existing equipment and services into integrated Product-Service System (PSS) solutions.

Creating new to the world products (artefacts) coupled with new services to operate within a new system requires management of higher amounts of solution space ambiguity than previously addressed in PSS design. Prototypes are a flexible vehicle of exploration for all the relevant disciplines to communicate across their boundaries and up the PSS value chain (Exner et al., 2016). Prototypes can take many different forms depending on their purpose. Whether it be to answer specific questions or explore solution and problem spaces, a well-designed prototype can act as an effective boundary object to achieve engagement across disciplines and stakeholders' boundaries (Bertoni et al., 2016). To develop these boundary object prototypes many elements and perspectives need to be balanced in order to realize maximum benefit from the resources invested. This balance requires the designer to be aware of and attentive to all the pertinent elements during the development and testing of the prototype.

Current support tools such as the 'prototyping canvas' are aimed to increase the designer's simultaneous cognition of these elements for product design (Lauff et al., 2019). Considering the additional elements persistent in radical PSS design, ticurrent designers need supplemental support to 
manage accelerated early phase ambiguity. In order to create a support tool for traditional equipment manufacturing companies to strategically design radical PSS solutions through prototyping it is necessary to map and understand how designers from the relevant disciplines view the what, why and when around prototypes and prototyping

The primary objective of this paper is to identify how practitioners responsible for design and development of products, services or systems currently utilize prototypes. These insights will be summarized and discussed with the intention for them to enable a deeper understanding of the gap between current practices and potential best practices. The ultimate purpose is to guide the creation of or identification of the appropriate available support tool(s).

\section{Scientific background}

\subsection{Ambiguity in practice}

Within the incumbent construction industry, designers and engineers in practice are less likely to encounter high levels of ambiguity. While some ambiguity exists more of what the designers/engineers encounter is uncertainty. Ambiguity refers to 'unknown unknowns' and Uncertainty is 'known unknowns'. This differentiation originates from Carleton et al.'s (2008) work on complex problem solving. Figure 1 visualizes in general how these two concepts change throughout the process although they do make clear that they fluctuate more discreetly than that graph reflects and that the phases overlap in practice.

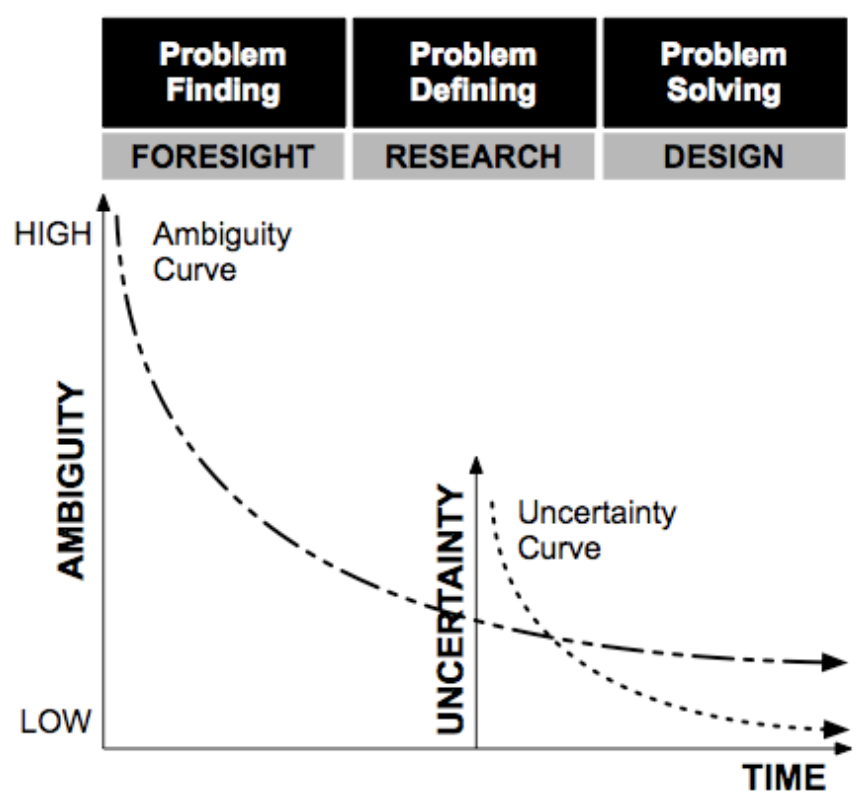

Figure 1. Uncertainty and Ambiguity Curve in complex problem solving (Carleton et al., 2008)

\subsection{PSS design and boundary object prototypes}

Within PSS design, boundaries exist between the required stakeholders and disciplines that must be overcome to attain a shared cognitive model for the concept and the customer perspective within the design team. Typically, attempts to address this challenge refer to artifacts or models that represent the concept as boundary objects. Djelassi and Decoopman (2016) define a boundary object as something that facilitates the exchange of knowledge and expertise between disparate actors in a network. Existing literature has highlighted the ability for prototypes to serve as effective boundary objects, but not all prototypes accomplish this goal due to the variety of prototyping methods available (Subrahmanian et al., 2003). Menold (2018) addresses the range of prototypes available to fit the desired function. Here they ground they process of prototyping into a systematized activity targeted at having a higher correlation between the artifact and the needs of the designer required to make the next decision. 
The state-of-the-art review in PSS design processes done by Rosa et al. (2017) showed the 14 most common process out of which only one used physical prototyping as an activity. Instead the processes all lean heavily on service blueprinting and frameworks for conveying concepts. The challenge here is that when we explore the Design Thinking (Leifer et al., 2011) literature, it heavily emphasizes the use of physical prototypes to convey the concept in a manner which has a higher rate of concept cognition especially when taking into account new to the world and radically different products and services.

This lines up with the desire to engage and enable humans in their exploration of the problem and solution space in order to produce the right solution for the right customer. This is in essence the core of PSS design, attempting to satisfy customer's needs in a manner that derives value beyond just the tangible product.

\section{Research approach}

The research presented in this paper can be framed as one of the activities conducted during the Descriptive Study I phase of the Design Research Methodology (DRM) proposed by Blessing and Chakrabati (2009). In this respect, interviews were identified to be a highly suitable method to answer the research questions related to the identification of the current as-is situation concerning utilization of prototypes. The interviews have been used to create and refine a reference model of the current status of the industry as it relates to the implementation of prototypes and identify themes to be addressed in a future PSS innovation support tool. Six interviews were conducted with five being employees of company A and one from company B. Company A was chosen due to their strides in innovation towards bold goals and are a rare actor in their industry with an emerging technology department exploring radical solutions. Company B was included since both the interviewee and the company have extensive experience in design and development of products across a broad spectrum. Neither of the companies are assumed to be atypical within their industry in the same manner as Herriott (2017). Interviewees were chosen specifically via intensity sampling which focuses on cases that strongly represent the phenomena of interest in this case because they represented different positions within the companies relevant to the design and development of products, services or systems. Each had at a minimum of five years' experience in their field (sans interviewee 5 in position 2 years) and a background with research around innovation and design thinking.

The results of the interviews were subjected to thematic coding via analysis of the interviews to identify divergences from literature prescribed prototyping practices and synthesis of the divergences into themes. This was conducted independently between two of the authors before the most relevant topics were converged upon together. This analysis and relation to the objective of the paper is organized by interview with specific quotes highlighting the identified themes. Each interview began with a short background discussion covering the research area context being PSS design to provide logical boundaries and guide the responses towards more relevant feedback. Then the interviewees were all asked the same following set of questions in a semi-structured interview process with more intricate dialog explored when appropriate:

1. How do you define a prototype? What are they? What are they used for?

2. When designing/developing new products \& services, how are the trade-offs identified and communicated between departments (internal stakeholders)?

3. How does a New Product/Service design project run? (what are the phases)

4. At what phases are prototypes used? How do they lead to improvements?

5. What methods are used to engage customers during the design phases?

\section{Results}

To present the data captured from interviews with conceptual coherence the results and analysis will be presented together. In this way, the discrete facts will better connect be grouped into comprehensible sub-patterns while simultaneously linking them to the overall objective proposed in this paper. To better guide the reader through the analysis Table 1 below highlights the key information brought up in each interview. 
Table 1. Interview highlights and breakdown

\begin{tabular}{|c|c|c|c|l|}
\hline Interviewee & Company & Current Role & Experience (years) & \multicolumn{1}{|c|}{ Theme(s) } \\
\hline 1 & A & Product & 15 & $\begin{array}{l}\text { Limited } \\
\text { Prototype } \\
\text { Applications }\end{array}$ \\
\cline { 3 - 5 } & & Designer/Engineer & & $\begin{array}{l}\text { Convergence } \\
\text { Prototypes only }\end{array}$ \\
\hline
\end{tabular}

Key Takeaways: Ambiguity encourages prototyping, but mainly for convergent verification/validation of features to avoid risk not divergent solution space exploration.

\begin{tabular}{|l|l|l|l|l|}
\hline 2 & B & $\begin{array}{c}\text { Product } \\
\text { Designer/Engineer }\end{array}$ & 10 & $\begin{array}{l}\text { Limited } \\
\text { Prototype } \\
\text { Application }\end{array}$ \\
\cline { 3 - 5 } & & & Ill-Defined \\
& & & $\begin{array}{l}\text { Prototype } \\
\text { Process }\end{array}$ \\
\hline
\end{tabular}

Key Takeaways: Tangible prototypes rarely used as boundary objects in multi-disciplinary design meetings, instead primarily CAD models or cost models inform decisions.

\begin{tabular}{|c|c|c|c|l|}
\hline 3 & B & $\begin{array}{c}\text { Product } \\
\text { Designer/Engineer }\end{array}$ & 10 & $\begin{array}{l}\text { Ill-Defined } \\
\text { Prototype } \\
\text { Process }\end{array}$ \\
\hline $\begin{array}{l}\text { Key Takeaways: Successful anecdotal projects drive individual design processes, fail to affect overall } \\
\text { prototype usage. }\end{array}$ & B & Innovation Manager & 8 & $\begin{array}{l}\text { Limited } \\
\text { Prototype } \\
\text { Application }\end{array}$ \\
\hline 4 & & & 8 \\
\hline
\end{tabular}

Key Takeaways: Good understanding of the best practices primarily applied to digital products instead of the larger products.

\begin{tabular}{|l|l|l|l|l|}
\hline 5 & B & $\begin{array}{c}\text { Service } \\
\text { Designer/Engineer }\end{array}$ & 2 & $\begin{array}{l}\text { Limited } \\
\text { Prototype } \\
\text { Application }\end{array}$ \\
\cline { 3 - 4 } & & & $\begin{array}{l}\text { Ill-Defined } \\
\text { Prototype } \\
\text { Process }\end{array}$ \\
\hline
\end{tabular}

Key Takeaways: Disconnect between designer and customers in service design process. Tangible prototypes only utilized with new to the company products.

\begin{tabular}{|c|c|c|c|l|}
\hline 6 & B & Innovation Manager & 8 & $\begin{array}{l}\text { Lack of New } \\
\text { Process } \\
\text { Adoption }\end{array}$ \\
\hline
\end{tabular}

Key Takeaways: Best practices concerning prototyping in design are encouraged, but changing official processes is cumbersome. Awareness of the ambiguity and available solution space breadth is limited.

\subsection{Interview 1 analysis}

This interviewee has been previously involved in small to medium organizations running product and service development for a wide range of products. Currently, they work at company B which operates as a middle-man between individuals and SME organizations desiring to take their product concepts through industrialization in preparation for mass manufacturing. The following quote reflects the bulk of the topic discussed in the interview relating to the overall design and development process:

$$
\begin{aligned}
& \text { "we've been doing design for years, but our official process and phases is continually } \\
& \text { changing" }
\end{aligned}
$$

They recognized having advantage as a smaller company more agile company when compared to larger companies with more rigid process flows. The interviewee continued to highlight over the course of the 
conversation how within an organization skills, capability, knowledge, and maturity all play a central role in when and why prototypes are created:

$$
\begin{aligned}
& \text { "we physically prototype certain interfaces or sub-systems until we know our } \\
& \text { modelling skills and knowledge have been validated and confirmed" }
\end{aligned}
$$

In the context of prototyping, an organization may utilize one strategy based on their current human and technological capability and organizational knowledge, but using each project as a learning opportunity, the rules for prototyping decisions should change. By rules, it was intended to mean the reasoning for the why to prototype. The term risk dominated the reasoning behind the bulk of decisions to go ahead with tangible prototypes. The final quote addresses each time a prototype is created, the organization's confidence in its ability to create a specific type of part or surface to reach certain physical requirement grows:

\section{"when you build hundreds of prototypes you get to know which things are going to work in the digital realm and which thing you have to build to get a sense of it. And some things you just have to build."}

This inversely affects the likelihood to prototype it in the future for the purposes of saving time and materials. Here a link can be made between ambiguity and a need for physical prototyping although not explicitly stated.

\subsection{Interview 2 analysis}

This interviewee has been part of an advanced engineering department for company for the last 10 years. Their role is that of a development engineer with broader responsibilities to explore various products beyond the scope of a single department. Having achieved their $\mathrm{PhD}$ in engineering, they also have a clear understanding of research and mechanical engineering. The first two quotes below reflect a common disconnection observed between their observed potential for prototypes to communicate across disciplinary boundaries and current perceptions of prototype form/function fit:

\section{"Physical Representations are the least commonly used in multidisciplinary design meetings" \\ "Testing/understanding for yourself or an audience" and "verification \& validation is more than a sketch or CAD, prototypes are tangible"}

A clear frustration was expressed when noting that no meetings that include other departments included prototypes, instead opting to base design decisions of the concepts by simplifying them into cost analysis on spread sheets or at best a CAD drawing. It shows how the mindset and organizational direction still lies heavily in late stage of production ready prototypes. The issue here is ignoring an entire arsenal of options for iterative development of final fidelity products. This omits the possibility of learning from early prototypes with the potential to uncover unaddressed design assumptions and potentially potent insight that could result in transformative customer satisfaction. During the interviewee's response to question 3 they revealed the following insight:

"We don't really know the formal process and don't really follow it because we work in the early, early phases before the formal process starts."

In the discussion they reiterated how they work primarily in the earliest phases of designing new concepts. More information concluded that the company's official development process is a stage-gate model which starts at the end of their conceptual phase where it is then handed off manufacturing for industrialization. Unfortunately, this conceptual work is not prescribed with any formal process, guidance or design support tools. Instead the company allows their highly experienced designer/engineer to utilize their skills creatively. Finally, the interviewee perceived themselves as working in the earliest stages of the design process, from a design thinking context their provided initial design prompts pre-emptively bound the design space. This limiting of ambiguity enables them to lean on previously successful ad-hoc methods familiar to the engineer instead of potentially more successful approaches. 


\title{
4.3. Interview 3 analysis
}

This engineer works at a facility in a different country than interviewee 2 that focuses on different equipment. Another critical difference is they are well versed in the language of innovation and design thinking resulting from being a current industrial $\mathrm{PhD}$ candidate in the area and working with innovation methods development with the emerging technologies department of the company. The following quote from the interview is pertinent to a specific topic:

\begin{abstract}
"for sure a prototype is a tool to support conveying a message or a new type of working on your system solution product in some way that people or the typical users can interact with your idea more than it is in the way that you just draw a picture or you have a sketch at hand or something like that."
\end{abstract}

This quote reflects a particularly interesting view on the power of physical vs two-dimensional (2D) prototyping or sketching. Expanding on this was a side conversation about whether CAD models count as $2 \mathrm{D}$ although they can be perceived as 3D. The interviewee's professional experience suggested nothing is 3D until it can be held and manipulated in your hands. Although if a CAD model was distributed to other engineers it could be interpreted as intended, but beyond the boundaries of the same discipline or department it served as more of a sketch needing direction to be correctly understood. Following are the additional quotes from the interview:

"We then utilize another way of working. So we bring all the different stakeholders together in one big open space office...we are bringing all people together in one open space office and there the engineers the aftermarket people manufacturing guys supply chain, they in purchasing they working all or sitting all together. So here it's more that they have all information available and they have short distances to the people having the information that is needed in each time step"

"They meet at the Prototype ... and one technician was more or less constantly there to manufacture or to bolt this stuff together. And so all these meetings had happened at that machine."

This situation being described is the company has had success in terms of demand from customers with a new machine concept unique to the market and want to capitalize quickly. The company concentrated all of the relevant internal stakeholders in one floor of a building primarily centred around a flexible prototype of the developing machine. With the quotes in context it can be inferred that there is a recognition of the value gained from having a tangible prototype enabling all internal stakeholders to more easily grasp the new concept and how it was evolving. Further information revealed a positive response from this department exhibited via reduction in time to make design decisions.

This described 'strategy' reflects more of a scrum mentality implemented to take advantage of an opportunity and enabled in this case because the machine was small enough to bring into an office building where all domain stakeholders are conveniently co-located. The primary takeaway is the company succeeded in building a prototype capable of facilitating concept cognition across disciplinary and departmental boundaries with positive effects. Another caveat, to address is in terms of innovation, is this project would not be considered radically different in overall function of the machine, but drastically different subsystems to accomplish this capability. Related to managing ambiguity this anecdotally successful scenario discussed in the interview is still also bounded at the existing machine level limiting the solution space.

\subsection{Interview 4 analysis}

This interviewee has been an Innovation manager at their previous company and in their current role in company A. Given their work and background they are versed in the academic prototyping language, producing a concise conversation with direct answers. The following quotes are the highlights from the interview:

"it's about utilizing the right, the appropriate prototype fidelity for the right purpose in order to get the right feedback" 
"They could envision how this would fit within their lifestyle or day-to-day and how that would provide value to them and they were asking when can I have it and that a completely different conversation"

These two quotes are in direct reference to the interviewee's experiences with tangible prototypes in their capacity with new concept development. The examples given were those of added functionality or sub-systems within a machine and particularly Human Machine Interfaces (HMI) systems. This respondent's capacity within their organization puts them in a unique position to be more frequently meeting customers and potential users of the under-development products. HMI systems are more portable again and software-based so the prototypes are more easily manipulated and changed than what other engineers may consider tangible or physical prototypes.

The primary take-aways from this interview are firstly, this was the only direct reference to the term fidelity, although it is a primary consideration during any prototyping activity. Secondly, the concept of "fit" is important to note because it has direct implications with respect to consideration of the various elements present in any prototype development. The final quote below reflects the discussion of partnerships with customers being invaluable in the new concept development process and even more when considering products, services and systems:

"if you don't work with partners, you are working in a black box and you will suffer the consequences for doing that ultimately when reality happens"

This mention of co-creation here is another vitally important strategy particularly when developing new to the world solutions as the customer input is a primary diver of the final value of a PSS solution.

\subsection{Interview 5 analysis}

The interviewee was a service designer at company A with nearly two years' experience in the position. Their primary responsibilities being to design additional services for new equipment. Verbiage used in the interview questions did not translate as well into this induvial's colloquial work language, so more stories were exchanged. The first quote is centred around one of the few times where a tangible prototyping entered their workflow:

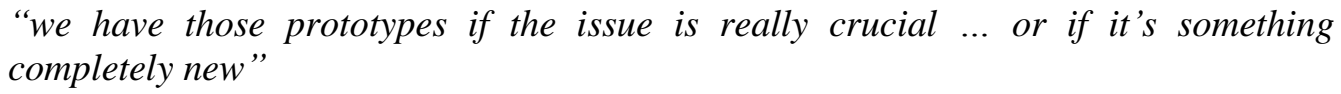

As a department, the interviewee's interaction with tangible prototypes only occurred when uniquely new or critical products were developed. This was done to understand the impacts and potential new (emergent) behaviors of the sub-system or machine level. The final two quotes are anecdotes summarizing a frustration originating from a lack of real interface with customers limiting empathy for how the overall solutions are perceived

"it can be really surprising actually to meet them and to hear their real-life experiences with the machine. It can be really surprising, but I would love to do it actually more often. I did it in my first year when I was a trainee"

"you develop something together and they see it in a completely different way. You spend time, like hours or days or weeks on something, then when they see it, they might say why did you do this?"

Within these narratives the interviewee highlights typically customer visits for service designers only occur once when they are being initially trained even though they left a positive impression. To further accentuate the issue, their final story showed an enduring perception of increased performance being misconstrued as a more desirable solution from the customer. This story consisted of investmenting hundreds of hours into the design and production of a better engineered filter solution before customer feedback being introduced. This resulted in the unveiling of the solution being rejected by customers due their trust in the previous solution even with the reduced performance. The overall sentiment conveyed in this interview is a disconnection between designers/engineers and their customers leading to a misconstruing value drivers in solutions at an early phase of design. 


\subsection{Interview 6 analysis}

This interviewee was in a role as an innovation manager and previously a department manager responsible for strategic innovation research projects at company A. This was a necessary perspective to gather as those responsible for managing innovation have a greater impact on strategies, methods and tools used during design and development than individual engineers or designers. This respondent has extensive experience working towards shifting the organizational mindset to be an innovation generator. During the interview the manager, who is also a researcher aware of best practices existing, they pointed out the current processes within the company limits the fostering of innovation. As exemplified in the following quote a primary barrier identified was an entrenched mindset of existing engineers:

\section{"... the question is also is it possible to change, can existing experienced engineers unlearn or is it that we need new types of engineers ...?"}

This mindset included a tendency to devalue customers as a source for input towards designing better machines and only producing prototypes as means to test physical characteristics with nearly complete final machines. The interviewee made it clear to delineate between prototyping for functional testing and prototyping for new innovative concepts. The following quotes coupled the company engineers' collective mindset on prototyping with their established process as an issue:

"The only problem is because of all those processes have the prototypes much too late and too much similar to the final product... you didn't allow yourself to question your prototypes enough..."

"it's almost like pre-production even with the first prototype. So, it's more to test the function than actually drive the conversation typically"

The need always exists to test and reduce risk before bringing equipment to market, but the newer mantra in innovation is the maker sure you're designing the right it before you design it right. This is where the concept of tackling ambiguity arose in the conversation as something engineers and designers trained in traditional PD methodologies have only dealt with in the area of feasibility. The interview concluded on the concept of how to support engineers and designers with massive amounts of tacit knowledge from experience in tackling the accelerating ambiguous challenges of coupled radical Products, services and systems.

\section{Discussion}

The interviews note a few particular themes to expand on when considering how and where strategies around the use of prototypes in practice can be supported to better address the accelerating ambiguity of radical PSS design and development.

Deriving from interviews and observations related to the practice of developing prototypes, there is a recurring theme concerning risk. The risks can be classified as potential failure modes of a physical product, unforeseen issues navigating an Human Machine Interface (HMI) in the machine, and/or safety concerns. These risks are identified well after ambiguous problems and solutions have been predetermined and reflect uncertainty of specific aspects of a well-defined concept (Carleton and Cockayne, 2009). This view results in each discipline or department building prototypes that allow them to assess the relevant criteria for them. This utilization of the prototyping mechanism is more restrictive than what is discussed in prototyping literature (Camburn, 2017). For instance, Menold et al.'s (2018) prototype for X framework emphasises flexibility in application and a fit between the desired knowledge and the prototype itself.

Interviewee 6, as a manager, perceived a lack of flexibility within the designers to grow their interpretation of prototypes and follow Camburn's (2017) cited research showing that the majority of prototypes should take place in the first 30\% of a project (Elsen et al., 2012). Interviews with the designers/developers themselves reflected an understanding of the expanded role of prototypes in the design process. The disconnect arose in how they presented their examples of prototypes not being used in design decision meetings. What they needed to build were boundary object prototypes for other 
departments and disciplines to be able to extract the same value from their prototypes. Support exists to augment this disconnect by Lauff (2019) in the form of a prototyping canvas. The canvas enables designers to incorporate the broader elements of a prototype of audience, material, driving questions, etc. By forcing the designer to think through these elements they are more informed with their choice of prototyping method resulting in better connection between the prototyping efforts and the questions answered.

As industry starts leveraging the technological revolution to transition towards radical solutions, current designers/engineers ability to manage ambiguity has been eroded over time through a focus on incremental improvement. As Norman and Verganti (2013) point out, focusing on the incremental innovation of the current hill (or 'known' solution) designers are less inclined or informed on how to seek alternate hills with higher potential radical innovation. Leifer et al. (2011) emphasise prototypes as a means for exploring early design phase ambiguity. Yet, a central narrative of the interviews revealed their dominant application in practice centred primarily on late stage verification and validation of physical performance. Even when designers/engineers perceived the prototypes they engaged with to be in an early phase it was clear problem/solution space boundaries had already been defined. This led to the interviewee's perceived applications for prototyping reflecting those successfully implemented in previous experiences. The omission of the explorative applications implied to the authors that in practice a narrower interpretation of a prototype's function compared to literature can occur (Menold et al., 2018; Camburn et al., 2017).

Stepping into the PSS frame beyond pure product development even more transdiciplinarity is needed to create these solutions. To date, the Prototyping Canvas does not address these additional elements of service and system. Adding these elements may result in an effective tool to support the creation of boundary crossing prototypes towards enabling designers/developers to better explore the added ambiguity they will face. It could be useful for future work to attempt a workshop centred around PSS design from which we may see differing results. However, this does not discount the observation that support for these activities is needed to ease the transition from one working mode to another.

From a methodological perspective the paper sees some limitation in relation to the number of interviews and lack of triangulation in data analysis. This is less relevant in qualitative research, it was more important that the interviewees represented highly relevant actors with experience in both their specialty and research around innovation. Nevertheless, the work contributed to build a clearer as-is description of the current situation by identifying critical challenges, issues, and requirements to enable the research process to enter an initial prescriptive study phase. In other words, the findings represent the first necessary knowledge to be used to generate initial support ideas. That knowledge will be further improved and updated in combination with more detailed investigations of the current situation, in line with the iterative nature of the DRM.

\section{Conclusions}

The objective of this paper was to identify where and how prototypes are being used in practice within a specific industry attempting to make a transition towards radically innovative PSS design. The resulting themes were set against the backdrop of literature-based guidance on the use of prototypes and radical innovation towards input for a future support tool. Concerning the concept of addressing ambiguity, it was clear that in practice the majority of designers are not typically currently participating in the phases of projects where the ambiguity was what literature would define as high. The counterintuitive aspect of this is that while the interviewees interpreted their own work as containing high levels of ambiguity, academics would more aptly define this as uncertainty. This contention between perspectives might lead designers/engineers with a lot of experience to retain a fixed mindset towards the use of prototypes.

Connecting all of the interviews, a common issue of addressing 'known unknowns' is persistent, but also solving these unknowns in known ways with little guidance on the breadth of methods and tools available. Currently, support tools such as the 'prototyping canvas' enable designers to better visualize the connections between prototype approach, features, needs and audiences for product development. To address the additional tangible and intangible components in future radical PSS design more elements need to be included. These additional elements should aim to address the broader stakeholder 
network and cross the boundaries of the growing number of disciplines required to holistically design/develop a PSS.

\section{Acknowledgments}

The authors would like to gratefully acknowledge the Knowledge Foundation and partners via the Model Driven Development and Decision Support project for their financials support Also, a sincere thanks to the industrial research partners contributing to the work.

\section{References}

Bertoni, A. and Bertoni, M. (2019), "Modeling 'ilities' in early product-service systems design", Procedia CIRP, Vol. 83, pp. 230-235. https://dx.doi.org/10.1016/j.procir.2019.03.091

Bertoni, M., Panarotto, M. and Larsson, T.C. (2016), "Boundary objects for PSS Design", Procedia CIRP. The Author(s), Vol. 47, pp. 329-334. https://dx.doi.org/10.1016/j.procir.2016.03.226

Blessing, L.T.M. and Chakrabarti, A. (2009), DRM, a Design Research Methodology.

Camburn, B. et al. (2017), "Design prototyping methods: state of the art in strategies, techniques, and guidelines", Design Science, Vol. 3 No. May, pp. 1-33. https://dx.doi.org/10.1017/dsj.2017.10

Carleton, T. and Cockayne, W. (2009), "The power of prototypes in foresight engineering", DS 58-6: Proceedings of ICED 09, the 17th International Conference on Engineering Design, Vol. 6 No. Part 2, pp. 267-276.

Carleton, T., Cockayne, W. and Leifer, L. (2008), "An exploratory study about the role of ambiguity during complex problem solving”, AAAI Spring Symposium - Technical Report, Vol. SS-08-03 No. May 2014, pp. 8-13.

Djelassi, S. and Decoopman, I. (2016), "Innovation through interactive crowdsourcing: The role of boundary objects", Recherche et Applications en Marketing (English Edition), Vol. 31 No. 3, pp. 131-152.

Elsen, C. et al. (2012), "Representation in early stage design: an analysis of the influence of sketching and prototyping in design projects", ASME 2012 International Design Engineering Technical Conferences and Computers and Information in Engineering Conference, Chicago, IL.

Exner, K. et al. (2016), "A transdisciplinary perspective on prototyping", 2015 IEEE International Conference on Engineering, Technology and Innovation/International Technology Management Conference, ICE/ITMC 2015, https://dx.doi.org/10.1109/ICE.2015.7438659

Herriott, R. (2017), Patient Involvement in Danish hospital design. Co-Design, (pp. 1-15). Published online. https://dx.doi.org/10.1080/15710882.2017.1292295

Lauff, C., Menold, J. and Wood, K.L. (2019), "Prototyping Canvas: Design Tool for Planning Purposeful Prototypes", Proceedings of the Design Society: International Conference on Engineering Design, Vol. 1 No. 1, pp. 1563-1572. https://dx.doi.org/10.1017/dsi.2019.162

Leifer, L.J. and Steinert, M. (2011), "Dancing with ambiguity: Causality behavior, design thinking, and tripleloop-learning", Information Knowledge Systems Management, Vol. 10 No. January 2011, pp. 151-173.

Menold, J., Simpson, T.W. and Jablokow, K. (2018), "The prototype for X framework: exploring the effects of a structured prototyping framework on functional prototypes", Research in Engineering Design, pp. 187-201.

Norman, D.A. and Verganti, R. (2013), "Incremental and Radical Innovation: Design Research vs. Technology and Meaning Change", Design Issues, Vol. 29 No. 4, pp. 1-5. https://dx.doi.org/10.1162/DESI

Rosa, M., Marques, C.A.N. and Rozenfeld, H. (2017), "Commonalities and Particularities of PSS Design Process and Design Thinking", Procedia CIRP. Elsevier B.V, Vol. 64, pp. 253-258. https://dx.doi. org/10.1016/j.procir.2017.03.020

Subrahmanian, E. et al. (2003), "Boundary objects and prototypes at the interfaces of engineering design", Computer Supported Cooperative Work (CSCW), Vol. 12 No. 2, pp. 185-203. 